\title{
Evidence for the Role of Histamine H3 Receptor in Alcohol Consumption and Alcohol Reward in Mice
}

\author{
Saara Nuutinen', Minnamaija Lintunen², Jenni Vanhanen', Tiia Ojala', Stanislav Rozov' and Pertti Panula*,' \\ 'Neuroscience Center and Institute of Biomedicine, Department of Anatomy, Biomedicum Helsinki, University of Helsinki, Helsinki, Finland; \\ ${ }^{2}$ Department of Biology, Abo Akademi University, Turku, Finland
}

\begin{abstract}
Recent research suggests that histamine $\mathrm{H} 3$ receptor $(\mathrm{H} 3 \mathrm{R})$ antagonism may diminish motivational aspects of alcohol dependence. We studied the role of H3Rs in alcohol-related behaviors using H3R knockout (KO) mice and ligands. H3R KO mice consumed less alcohol than wild-type (WT) mice in a two-bottle free-choice test and in a 'drinking in the dark' model. H3R antagonist ciproxifan suppressed and H3R agonist immepip increased alcohol drinking in C57BL/6J mice. Impairment in reward mechanisms in H3R KO mice was confirmed by the lack of alcohol-evoked conditioned place preference. Plasma alcohol concentrations of H3R KO and WT mice were similar. There were no marked differences in brain biogenic amine levels in $\mathrm{H} 3 \mathrm{R}$ KO mice compared with the control animals after alcohol drinking. In conclusion, the findings of this study provide evidence for the role of $\mathrm{H} 3 \mathrm{R}$ receptor in alcohol-related behaviors, especially in alcohol drinking and alcohol reward. Thus, targeting H3Rs with a specific antagonist might be a potential means to treat alcoholism in the future. Neuropsychopharmacology (20I I) 36, 2030-2040; doi:I0.1038/npp.201 I.90; published online 8 June 20I I
\end{abstract}

Keywords: alcohol use disorders; alcoholism; drinking in the dark; $\mathrm{H} 3$ receptor; histamine

\section{INTRODUCTION}

Brain histaminergic neurons are localized in the tuberomamillary nucleus of the posterior hypothalamus and send both ascending and descending projections to most parts of the brain (Haas and Panula, 2003). Histamine $\mathrm{H} 3$ receptor (H3R) is one of the four receptors (H1-H4) mediating the effects of neuronal histamine. H3R is highly expressed in the brain and it differs from other histamine receptor types by its exceptionally multifunctional role. H3R was cloned in 1999 (Lovenberg et al, 1999) but already in the beginning of the 1980s it was characterized as a typical autoreceptor, which regulates the release and synthesis of histamine (Arrang et al, 1983, 1987). Later it was found that H3R functions also as a heteroreceptor, which regulates the release of many other neurotransmitters, such as dopamine, acetylcholine, noradrenaline, and GABA (Haas and Panula, 2003; Schlicker et al, 1994). These properties of H3Rs initiated a broad interest within the pharmaceutical industry to develop specific H3R ligands for the treatment of, for example, sleep-wake disorders and obesity (histamine-dependent) and attention and cognitive deficits (noradrenaline- and acetylcholine-dependent). More recently, it was shown that $\mathrm{H} 3 \mathrm{R}$ is expressed also at

\footnotetext{
*Correspondence: Professor P Panula, Neuroscience Center and Institute of Biomedicine, Anatomy, POB 63, 00014 University of Helsinki, Helsinki, Finland, Tel: +3589 1912 5263, Fax: + 3589 1912 5261, E-mail: pertti.panula@helsinki.fi Received I5 February 2011; revised 4 April 2011; accepted 27 April 2011
}

postsynaptic sites (Pillot et al, 2002, 2003). H3R was found to be highly expressed in the striatum on GABAergic cell bodies of the medium spiny neurons. Interestingly, these postsynaptic H3Rs are able to form functional heterodimers with dopamine D1 and D2 receptors in vitro and in vivo which integrate histamine- and dopamine-related signals (Ferrada et al, 2008, 2009; Moreno et al, 2011). Further, H3R receptors display high constitutive activity, which adds to the complex functional role of this receptor type (Arrang et al, 2007). The high expression level of $\mathrm{H} 3 \mathrm{R}$ in the mesolimbic system and the ability of H3Rs to affect brain dopaminergic functions via regulation of dopamine release and interaction with postsynaptic dopaminergic receptors raise a question whether H3Rs could be involved in brain reward processes underlying the development of addictive behaviors.

Recent reports suggest that $\mathrm{H} 3 \mathrm{R}$ indeed has a role in the regulation of substance abuse-related behaviors (Panula and Nuutinen, 2011; Brabant et al, 2010). Treatment with H3R antagonists suppresses alcohol drinking in alcohol-preferring rats (Lintunen et al, 2001; Galici et al, 2010) and inhibits alcohol reward in a conditioned place preference (CPP) paradigm in mice (Nuutinen et al, 2010a). The H3R antagonists themselves are not rewarding or aversive (Nuutinen et al, 2010a; Munzar et al, 2004). H3R antagonists modulate also the effects of other drugs such as cocaine (Brabant et al, 2005, 2006, 2009; Clapham and Kilpatrick, 1994) and amphetamine (Munzar et al, 1998, 2004). However, one of the probable mechanisms underlying the changes in cocaine responses might be the interaction of the imidazole-based H3R antagonists with 
the liver cytochrome P-450 enzymes resulting in higher concentration of cocaine in the blood (Brabant et al, 2009).We have not detected H3R ligand-induced alterations in plasma alcohol concentrations suggesting that H3R antagonism could be beneficial in the treatment of alcoholism.

Our previous studies suggest that brain histaminergic system may also have a role in the motor impairment by alcohol. Mice lacking histamine synthetizing enzyme, histidine decarboxylase are not stimulated by alcohol (Nuutinen et al, 2010a) and H3R antagonists increase the stimulatory response by alcohol in DBA/2J mice (Nuutinen et al, 2010b) and decrease it in C57BL/6Sca mice (Nuutinen et al, 2010a). Further, rats with high tolerance to alcohol show impaired performance on a tilting plane motor function test in response to $\alpha$-fluoromethylhistidine, a suicide inhibitor of histidine decarboxylase (Lintunen et al, 2002).

Limited data are available about brain histaminergic system in human alcoholics. Histamine levels in cortical grey matter were higher in type 1 alcoholics (late onset, often females, low degree of association with violence) than in normal control brains (Alakarppa et al, 2002). The levels of the first metabolite of histamine, tele-methylhistamine, were significantly increased in type 2 alcoholics (early onset, often males, high degree of association with violence) indicating increased histamine release and turnover (Alakarppa et al, 2002). This may mean that histamine synthesis and/or metabolism are primarily altered in alcoholics, or that the possibly associated liver pathology with increased blood L-histidine lies behind the abnormal findings. In agreement with the human data, the alcohol-preferring AA rats (Alko, alcohol) display high brain histamine concentrations and increased density of histaminergic fibers in comparison with control Sprague-Dawley rats or alcohol non-preferring rats (Lintunen et al, 2001).

Despite the recent findings that support a role for H3Rs in the modulation of alcohol-related behaviors, targeting brain histaminergic system is not commonly listed as a strategy to treat alcoholism. In this study, we tested the hypothesis that $\mathrm{H} 3 \mathrm{R}$ has a role in alcohol consumption in mice by using H3R knockout (KO) animals and using specific H3R ligands in wild-type (WT) mice. On the basis of our previous findings with $\mathrm{H} 3 \mathrm{R}$ drugs, we also hypothesized that $\mathrm{H} 3 \mathrm{R} \mathrm{KO}$ mice may display altered alcohol-induced CPP. Third, we examined whether H3R gene deletion affects alcohol-evoked locomotor activity and motor impairment. As hypothesized, we found alterations in all of these behaviors tested in H3R $\mathrm{KO}$ mice and detected marked changes in alcohol consumption after treatment with H3R ligands. To test whether changes in neurotransmitter levels could explain the findings in $\mathrm{H} 3 \mathrm{R} \mathrm{KO}$ mice, brain tissue contents on biogenic amines were studies. However, no dramatic changes were found between H3R KO and WT mice.

\section{SUBJECTS AND METHODS}

\section{Animals}

Male H3R KO (backcrossed to the C57BL/6J strain and identified as H3R KO, (Toyota et al, 2002) mice were supplied by Johnson and Johnson Pharmaceutical Research and Development, LLC, La Jolla, CA, bred and maintained by the Jackson Laboratory, which also supplied the WT C57BL/6J control mice. The H3R KO mice were generated on a background of 129/Ola and C57BL/6J mice. Selective backcrossing (10 times) followed by microsatellite marker analysis was conducted to generate a strain, which has at least $99.5 \%$ genetic identity to C57BL/6J mice (Toyota et al, 2002). For the drinking in the dark (DID) trial where the effects of H3R ligands on alcohol drinking were tested, JAX C57BL/6J were ordered from Charles River (France). The JAX C57BL/6J strain is equivalent in genetic quality to those bred by the Jackson Laboratory. Thus, all the mice in this study were of the same C57BL/6J genetic background. The total number of animals used in this study was 165 .

Animals were housed in groups of 4-5 with the exception of alcohol drinking experiments where mice were single housed. Standard food pellets (Scanbur, Sweden) and water were available ad libitum in the home cage. The animal room was maintained on a $12-12 \mathrm{~h}$ light-dark cycle (lights on at 0600 hours) except in the DID experiment where reverse light cycle was used. Temperature and humidity were controlled at $20 \pm 1{ }^{\circ} \mathrm{C}$ and $50 \pm 10 \%$, respectively. Experiments were carried out during the light phase between 0700 and 1300 hours with the exception of DID study. The training of the mice for the rotarod and balance beam task was conducted between 0800 and 1600 hours. The principles of the Finnish Act on the Use of Animals for Experimental Purposes were followed in conducting these studies and the protocols were approved by the Animal Experiment Committee of the State Provincial Office of Southern Finland and by the Institutional Animal Care and Use Committee of Abo Akademi University.

Animals were brought to the experiment room 30-40 min before each trial to let them habituate to the change of an environment. The mice in alcohol drinking, place preference, and locomotor activity studies were drug naïve. The mice that were used in the CPP experiment were used in the balance beam experiment after a recovery period of 7 weeks. Two WT mice were excluded from the balance beam experiment because of unexpected health problems. The mice that were in locomotor activity studies were later used in the rotarod experiment after a 3-week drug-free period. The mice from the two-bottle choice test were used for the measurement of loss of righting reflex (LORR). Neurotransmitter and alcohol concentrations were measured from mice that were in behavioral experiments.

\section{Drugs}

Alcohol drinking solutions were prepared from $99.5 \%$ alcohol solution (Altia, Rajamäki, Finland) and diluted into $3-20 \%$ solutions (v/v) using tap water. Saccharin $(0.033$ and $0.066 \% \mathrm{w} / \mathrm{v})$, quinine $(15$ and $30 \mu \mathrm{M})$, and sucrose $(10 \%$, $\mathrm{w} / \mathrm{v})$ were dissolved in tap water. Alcohol for injections was diluted to $20 \%(\mathrm{w} / \mathrm{v})$ with sterile saline $(0.9 \% \mathrm{NaCl})$. Ciproxifan hydrochloride (Sigma-Aldrich, St Louis, MO), immepip dihydrobromide (from Professor Rob Leurs, Vrie Universiteit, Amsterdam, The Netherlands) were dissolved in saline. All drug doses correspond to the free bases of the drugs. All drug injections were given intraperitoneally with an injection volume of $0.01 \mathrm{ml} / \mathrm{g}$. 


\section{Alcohol Drinking - Two-Bottle Free Choice}

To assess alcohol self-administration and preference the animals were single-housed and trained to drink in a twobottle choice procedure. In total, the experiment lasted for 14 weeks. First, mice were given 1 week to habituate to two water bottles. Then animals were given $24 \mathrm{~h}$ access to two bottles, one containing plain tap water and the other containing an alcohol solution. The concentration of alcohol was raised every fourteenth day, increasing from 3 to 6 to 10 and to $20 \%$. The consumption of alcohol and water and animal weight was measured four times for each alcohol concentration with 2- to 5-day intervals. The positions of the bottles were changed at the same time with fluid consumption measurements. The potential differences in taste preference were also examined. Two weeks after the alcohol self-administration procedure, the same mice were tested for saccharin (sweet, no caloric value) and quinine (bitter) fluid intake and preference by offering first 0.033 and $0.066 \%$ saccharin solutions both for 1 week. After a recovery for 1 week with two bottles of water, the mice were given 15 and $30 \mu \mathrm{M}$ quinine solutions both for 1 week. The saccharin and quinine solutions consumptions were determined for each concentration twice with 2-day intervals. Throughout the experiment, fluid intake and body weight were measured using an analytical balance with a precision of $0.1 \mathrm{~g}$. During the alcohol self-administration experiment, average alcohol consumption per day per body weight was calculated for each measurement. The density of alcohol $(0.7894 \mathrm{~g} / \mathrm{l})$ was taken into account in the calculations. The average of the four measurements for each mouse was used in the final analysis. A measure of relative alcohol preference was calculated for each alcohol concentration by dividing the alcohol consumption by the total fluid (alcohol plus water) consumption. Similarly, relative taste preference was calculated at each concentration by dividing the saccharin or quinine solution consumption by total fluid consumption.

\section{Drinking in the Dark}

The consumption of alcohol in $\mathrm{H} 3 \mathrm{R}$ KO mice and the effect of H3R ligands in C57BL/6J mice were studied using the DID procedure with minor modifications (Rhodes et al, 2005). The light-dark cycle was reversed 2 weeks before the experiment and mice were single-housed for 1 week before the beginning of the DID sessions. In brief, starting $3 \mathrm{~h}$ after lights shut off, the water bottles were replaced with a $10 \mathrm{ml}$ graduated cylinder fitted with a double-ball- bearing sipper tube (to prevent leakage) containing $20 \%(\mathrm{v} / \mathrm{v})$ alcohol and left in place for 2 or $4 \mathrm{~h}$. Control animals received $10 \%(\mathrm{w} / \mathrm{v})$ sucrose. When studying the H3R KO mice and their WT controls, the mice received alcohol or sucrose 12 times during a period of 2.5 weeks (weekends off). Rhodes et al (2005) have previously shown that the alcohol drinking of C57BL/6J mice remains stable despite the weekend breaks. In the DID trial in which the effects of H3R ligands on alcohol drinking were tested, mice were given access to alcohol or sucrose four times a week on Mondays, Tuesdays, Thursdays, and Fridays for 3 weeks. The first week served as a habituation week. During the second week, the mice were injected with ciproxifan or immepip 30 min before giving access to alcohol or sucrose. A within-subject design was used so that each animal received three different doses of the drug and the saline control injection. The order in which the four injections were administered was computer-randomized so that each mouse received the injections in a different order. The injections were given on Tuesdays or Fridays. The rationale for allowing the mice to experience the drinking solutions without injections on alternate days was to reduce the chance that a taste aversion might develop from always pairing a drinking solution with an injection. The amount of alcohol consumed (volume) was recorded immediately after each drinking session and converted to grams per kilogram using each animal's alcohol consumption and body weight.

\section{Alcohol-Induced CPP}

CPP paradigm was used as described in (Nuutinen et al, 2010a) and it followed the principles of an unbiased, fully counterbalanced conditioning described by (Cunningham et al, 2006). Conditioning cages were individual transparent plastic cages $(42 \times 26 \times 20 \mathrm{~cm})$ covered with clear plastic lids with ventilation holes. Copper grid and plastic bathroom carpet material were used as conditioning cues on the cage floors. The trial lasted for 3 weeks and consisted of three phases: habituation (one session), conditioning (12 sessions), and preference testing (two sessions). The activity of mice was recorded in each phase (habituation, conditionings, and preference test) using video camera attached to Ethovision Color-Pro 3.0 video-tracking software (Noldus Information Technology, Wageningen, The Netherlands).

Habituation (day 1). Animals were weighed and given a saline injection just before placement in the center of the conditioning cage (no extra floor materials) for $5 \mathrm{~min}$.

Conditioning (days 2-13). Mice were randomly assigned to one of the two conditioning subgroups (Grid + or Grid-). Mice in the Grid + condition received alcohol ( $2 \mathrm{~g} / \mathrm{kg}$, i.p.) paired with metal grid floor and saline paired with the plastic floor. Mice in the Grid- condition received alcohol paired with the plastic floor and saline paired with the metal grid floor. Each animal received six 5-min conditioning trials of each type on alternating days. The order of alcohol and saline exposure was counterbalanced within groups.

Place preference test (day 14). The preference test was carried out $24 \mathrm{~h}$ after the last conditioning trial. Immediately after saline injection mice were placed in the center of the apparatus with both test floors (half metal grid/half plastic material). The left-right position of the floors was counterbalanced within groups. The time spent during $15 \mathrm{~min}$ in different zones of the cage (metal grid or plastic floor) and the total distance moved were recorded. Time spent on the grid floor was used as a primary dependent variable in data analysis. 


\section{Effect of Alcohol on Locomotor Activity}

The stimulating and depressant effect of alcohol in H3R KO mice was examined using locomotor activity recordings. The mice were then put to the plastic test cages without any additional floor materials. The $90-$ min habituation period was used to exclude the effect of explorative activity on the drug treatments. After the habituation period animals received an alcohol injection (1.5 or $2.5 \mathrm{~g} / \mathrm{kg}$, i.p.) after which they were immediately put back to the test cage. Activity of the mice was recorded by using a video camera and Ethovision Color-Pro 3.0 video-tracking software.

\section{Balance Beam}

The mice were trained to walk along a beam $(100 \mathrm{~cm}$ long, $1.5 \mathrm{~cm}$ diameter) four times per day on two separate sessions. All mice learned this task in 4 days. On the day of the experiment, the mice were given an alcohol injection $(1.2 \mathrm{~g} / \mathrm{kg}$, i.p.) $5 \mathrm{~min}$ before putting the mice on the balance beam. The behavior of the mice was recorded by a digital video camera and the number of footslips was assessed after the experiment from the recorded video.

\section{Rotarod}

The mice were trained six times per day in two separate sessions for 4 days to stay on the rotating $3 \mathrm{~cm}$ diameter rod (Rotarod, Ugo Basile, 47600, Comerio, Italy). During the training period, the acceleration of the rotatod was set from 5 to 40 rounds per min (r.p.m.), reaching the maximum speed at $3 \mathrm{~min}$. On the day of the experiment, the baseline performance was assessed by using acceleration 5-30 r.p.m. All mice stayed on the rotarod for at least $140 \mathrm{~s}$, which was then set to be the baseline performance. The alcohol injections were given cumulatively $(1.5 \mathrm{~g} / \mathrm{kg}$ plus and $0.5 \mathrm{~g} / \mathrm{kg}$ ) with a 15 -min interval $10 \mathrm{~min}$ before putting the mice on the rotarod. The motor performance was quantified as latency to fall (s).

\section{Loss of Righting Reflex}

H3R KO mice from the two-bottle choice experiment were used to study the LORR by alcohol after a 4-week recovery period. A sedative dose of alcohol $(4.0 \mathrm{~g} / \mathrm{kg}$, i.p.) was injected and the time of LORR (LORR latency, onset of sedation; inability of mice to right themselves when positioned on their back) and the time to regain it (LORR duration, time elapsed between the onset of sedation and righting of mice back on all four paws) were recorded. Recovery was defined as the time at which mice could right themselves three times in $30 \mathrm{~s}$ after being placed on their backs.

\section{HPLC Analysis of Biogenic Amines}

Brain samples for the analysis of biogenic amines were collected in the end of the DID study. Mice were killed with $\mathrm{CO}_{2}$ and decapitated. Prefrontal cortex samples were cut with a razor blade (approximately $+2.0 \mathrm{~mm}$ from Bregma) and the olfactory bulbs were dissected and discarded. Striatal samples including the nucleus accumbens were collected with forceps. Samples were frozen on dry ice and stored at $-80^{\circ} \mathrm{C}$ until analyzed. For the analysis the brain samples were thawed, weighed and sonicated on ice in 10 volumes of $0.3 \mathrm{M}$ perchloric acid, followed by centrifugation for $30 \mathrm{~min}$ at $15000 \mathrm{~g}$ at $+4^{\circ} \mathrm{C}$. Samples were then filtered through $0.45 \mu \mathrm{m}$ PVDF Acrodisk syringe filters (Pall Life Sciences, Ann Arbor, MI) and stored at $-80^{\circ} \mathrm{C}$ until determination. The concentrations of dopamine, DOPAC (3,4-dihydroxyphenylacetic acid), HVA (homovanillic acid), 5-HT, 5-HIAA (5-hydroxy-indoleacetic acid), NA (noradrenaline), and MHPG (3-methoxy-4-hydroxyphenylglycol) were measured with a chromatographic system that consisted of a Waters 515 pump (Waters Corporation, Milford, MA), Waters 717 Plus autosampler, Waters Concorde electrochemical detector equipped with a glassy carbon $3 \mathrm{~mm}$ electrode and was controlled by Waters Millenium software package. The SecurityGuard C18 $4 \times 3 \mathrm{~mm}$ precolumn (Phenomenex), column Phenomenex Gemini C18 $4.6 \times 1505 \mu \mathrm{M}$ and the flow cell were thermostated at $+35^{\circ} \mathrm{C}$. Electrode potential was set at + $0.8 \mathrm{~V}$. Mobile phase consisted of $50 \mathrm{mM}$ citric acid, $1.5 \mathrm{mM}$ 1-octanesulfonic acid sodium salt, $0.05 \mathrm{M}$ EDTA, 8\% methanol and $50 \mathrm{mM}$ o-phosphoric acid, and $\mathrm{pH}$ was adjusted to 2.7. The analysis was performed at a flow rate of $1 \mathrm{ml} / \mathrm{min}$ in isocratic mode.

\section{Plasma Alcohol Concentrations}

Plasma alcohol concentrations were determined in H3R KO and WT mice after acute alcohol administration $(2.0 \mathrm{~g} / \mathrm{kg}$, at $5 \mathrm{~min}$ ) and following 4-h DID session. In addition, alcohol concentrations were measured from those WT mice that received H3R ligand before DID. Terminal blood samples were collected via cardiac puncture (with a $1 \mathrm{ml}$ syringe and a $20 \mathrm{G}$ needle) immediately after euthanizing the mouse with $\mathrm{CO}_{2}$. Blood samples were transferred to cold lithiumheparin tubes and centrifuged at $2000 \mathrm{~g}, 2 \mathrm{~min}$. Plasma samples were transferred to microcentrifuge tubes and kept at $-80^{\circ} \mathrm{C}$ until analyzed. A commercial enzyme-based assay (Abcam, ab65343, Cambridge, UK) was used for the determination of alcohol concentration of the plasma samples.

\section{Statistical Analysis}

Statistical analysis was done with one-, two-, or three-way analysis of variance (ANOVA) combined with appropriate post-tests (Tukey or Bonferroni). Repeated-measures (RM) ANOVA was used in the analysis of the two-bottle choice, DID, and rotarod results. T-test was used for the analysis of neurotransmitter and alcohol concentrations. Values exceeding more than two SD from the group mean (eg, because of leakage of the drinking tubes) were excluded.

\section{RESULTS}

H3R KO Mice Drink Less Alcohol in a Two-Bottle FreeChoice Test

In a two-bottle free-choice protocol in which mice could choose either water or an ascending series of alcohol concentrations $(3,6,10$, and $20 \%)$, the $\mathrm{H} 3 \mathrm{R} \mathrm{KO}$ animals 

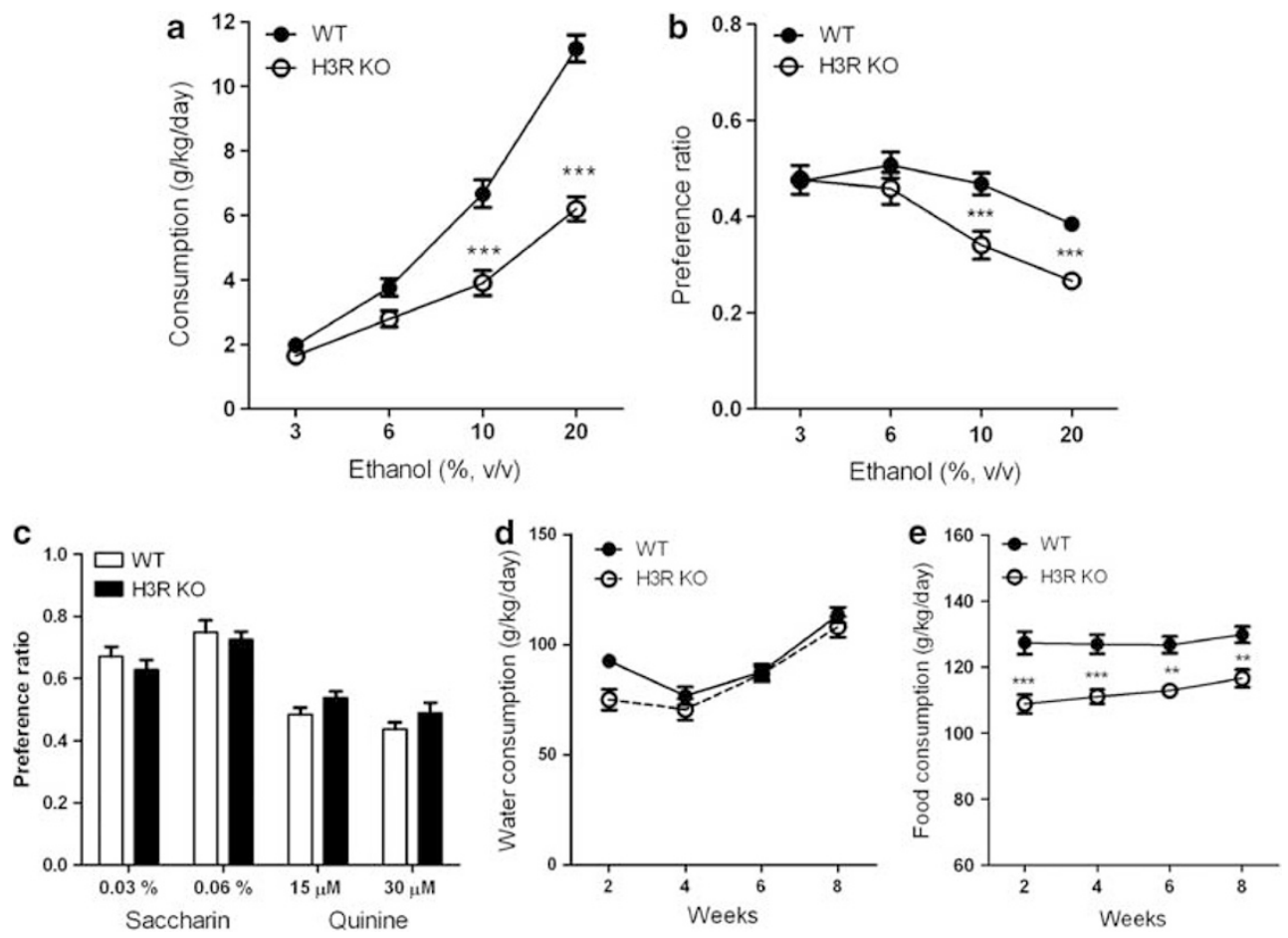

Figure I Alcohol consumption, preference, taste preference, water, and food consumption in H3R KO. Alcohol consumption (a) and preference ratio (b) of $\mathrm{H} 3 \mathrm{R} \mathrm{KO}$ mice in a two-bottle choice experiment. No differences in taste preference (c) for saccharin (sweet) and quinine (bitter) or in water consumption (d). The food consumption (e) is significantly lower in H3R KO mice compared with the controls. All data are expressed as mean \pm SEM. $n=10$ for both genotypes. ${ }^{*} * P<0.01$; ${ }^{*} * * P<0.001$, two-way ANOVA and Bonferroni post-test.

consumed significantly less alcohol $(\mathrm{g} / \mathrm{kg}$ of body weight per day) than their WT controls confirmed by a significant genotype $\times$ alcohol concentration interaction with RM twoway ANOVA $\left(\mathrm{F}_{3,54}=27.47, P<0.0001, n=10\right.$, Figure 1a). Bonferroni post-test revealed that the H3R KO mice drank significantly less 10 and $20 \%$ alcohol solutions $(P<0.01$ for both concentrations). A significant genotype $\times$ alcohol concentration interaction was found also for the alcohol preference $\left(\mathrm{F}_{3,54}=4.99, P=0.0040\right.$, Figure $\left.1 \mathrm{~b}\right)$ with significant differences at 10 and $20 \%$ concentrations (both $P<0.01)$ revealed by post-test. There were no significant differences in saccharin $\left(\mathrm{F}_{1,18}=0.10, P=0.7574\right)$ or quinine $\left(\mathrm{F}_{1,18}=0.00, P=0.9773\right)$ preference between H3R KO and WT mice (Figure 1c) suggesting that alcohol avoidance of H3R KO mice is not due to taste neophobia. Water consumption of the two genotypes was not significantly different throughout the experiment (Figure 1d). The food consumption of H3R KO mice was found to be significantly lower compared with the WT mice indicated by a significant genotype effect $\left(F_{1,18}=22.04\right.$, Figure 1e).

\section{H3R KO Mice Drink Less Alcohol in a Binge Drinking Model}

We then measured the consumption of alcohol in H3R KO and control C57BL/6J mice after 2 and $4 \mathrm{~h}$ in a limited access binge drinking paradigm (Figure 2). H3R KO mice consumed significantly less alcohol at both time points indicated by significant genotype effects in two-way ANOVA ( 2 h: $\mathrm{F}_{1,210}=32.46, P<0.0001 ; 4$ h: $\mathrm{F}_{1,198}=93.44$, $P<0.0001, n=10$, Figures $2 \mathrm{a}$ and $\mathrm{b}$ ). However, Bonferroni post-test only revealed significantly different time points for the 4-h drinking data. There was also a significant difference between the genotypes in average daily alcohol intake at 2 and $4 \mathrm{~h}(P<0.0001$ for both time points, $t$-test, Figure $2 \mathrm{c})$. The consumption of sucrose was found to be lower in H3R $\mathrm{KO}$ mice than in control mice indicated by significant genotype effects: $2 \mathrm{~h}: \mathrm{F}_{1,168}=50.90, \quad P<0.0001 ; \quad 4 \mathrm{~h}$ : $\mathrm{F}_{1,167}=93.74, P<0.0001$, Figures $2 \mathrm{~d}$ and e). There was also a significant time effect for sucrose drinking $(2 \mathrm{~h}$ : $\left.\mathrm{F}_{11,168}=12.55, P<0.0001 ; 4 \mathrm{~h}: \mathrm{F}_{1,167}=15.97, P<0.0001\right)$. Average daily consumptions were significantly lower in H3R KO mice $(P<0.0001$ for both time points, one-way ANOVA, Tukey's post-test, Figure 2f).

\section{Binge Drinking is Suppressed by H3R Antagonist Treatment}

To determine whether H3R antagonist ciproxifan (acting as an inverse agonist) can inhibit alcohol DID, mice were treated with $0.3,1$, or $3 \mathrm{mg} / \mathrm{kg}$ ciproxifan before alcohol drinking session. Ciproxifan in each given dose significantly reduced the volume of alcohol consumed as compared with the saline-treated mice $(\mathrm{F}=19.16, P<0.0001$, $\mathrm{RM}$ two-way ANOVA, $n=12$, Figure $3 a$ ). In contrast, ciproxifan injections had no effect on sucrose intake $(\mathrm{F}=1.754, P=0.1773$, $n=11$, Figure $3 \mathrm{~b}$ ). In line with these findings, the $\mathrm{H} 3 \mathrm{R}$ agonist immepip did not alter sucrose intake $(F=0.3905$, $P=0.7607, n=10$, Figure $3 \mathrm{~d}$ ), but significantly increased alcohol drinking with the $30 \mathrm{mg} / \mathrm{kg}$ dose $(\mathrm{F}=6.701$, $P=0.01, n=12$, Figure $3 c$ ). 

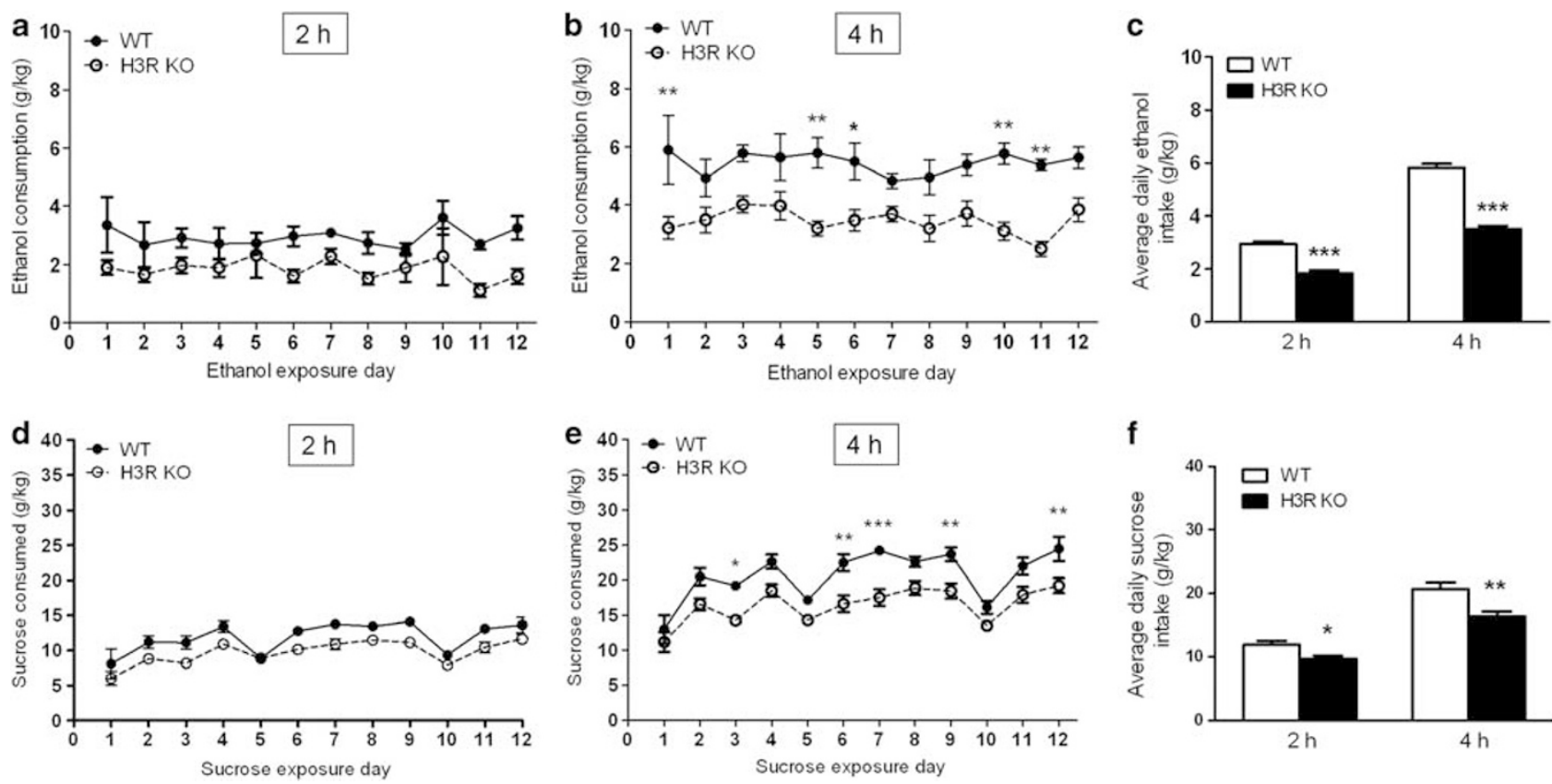

Figure 2 Alcohol and sucrose consumption in a limited access binge drinking model in $\mathrm{H} 3 \mathrm{R} \mathrm{KO}$ and control mice. $\mathrm{H} 3 \mathrm{R} \mathrm{KO}$ mice drank less alcohol than their control C57BL/6) mice during 2 (a) and 4h (b) of DID. There are significant genotype effects by two-way ANOVA: $2 \mathrm{~h}: P<0.000$ I; 4 h: $P<0.000 \mathrm{I}$, $n=10$ per group. However, Bonferroni post-test revealed significantly different time points only for the 4-h drinking data, $* P<0.05$, $* * P<0.01$. There was also a significant difference in the average daily alcohol consumption (c), **** $<<0.000$ I, one-way RM ANOVA, Tukey's post-test. Sucrose consumption was also significantly lower in $\mathrm{H} 3 \mathrm{R} \mathrm{KO}$ mice than in controls (d, e) indicated by significant genotype effects $(P<0.000 \mathrm{l}$ for both 2 and $4 \mathrm{~h})$. Average daily consumptions of sucrose ( $f$ ) were significantly lower in H3R KO mice $(P<0.000$ I for both time points, one-way RM ANOVA, Tukey's post-test).
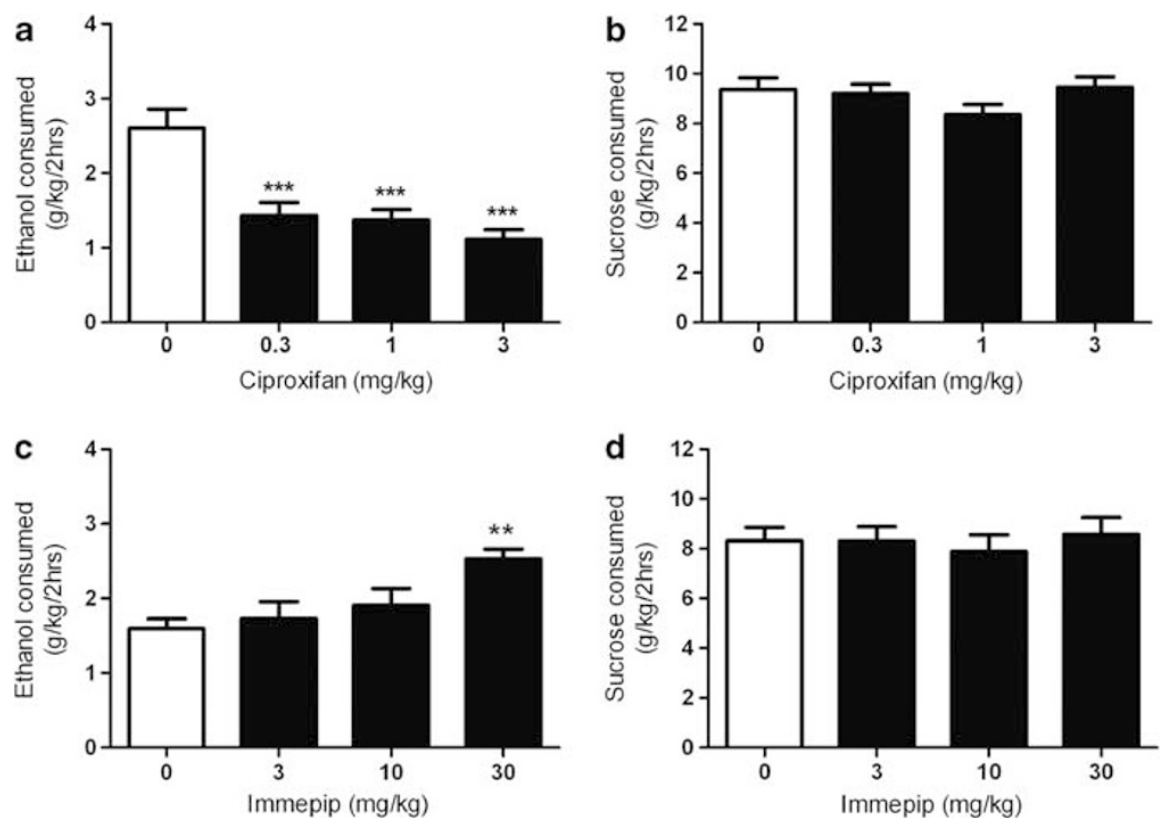

Figure 3 Histamine H3 receptor antagonist inhibits and agonist increases alcohol DID in C57BL/6) mice. (a) Bar graph representation of total alcohol intake over the 2-h DID assay (g/kg) for 0 (saline), 0.3, I, or $3 \mathrm{mg} / \mathrm{kg}$ ciproxifan preinjection. (b) Total sucrose volume intake after an i.p injection of saline or one of the three ciproxifan doses. (c) Total volume of alcohol intake after saline, 3, 10, or $30 \mathrm{mg} / \mathrm{kg}$ immepip injection. (d) Sucrose intake following a preinjection of saline or immepip. Data are presented as mean + SEM. *** $P<0.0$ I, ****P $<0.00$ I compared with the saline controls, one-way RM ANOVA,

Tukey's post-test, $n=10-13$ per group.

\section{Lack of Alcohol Reward and Reinforcement in H3R KO Mice}

The rewarding and reinforcing properties of alcohol in H3R KO mice were tested in a CPP paradigm with six conditioning sessions with alcohol and six sessions with saline on alternate days. When comparing the activity of mice during conditioning sessions three-way ANOVA revealed significant effects for time $(F=27.9, P<0.0001)$, 

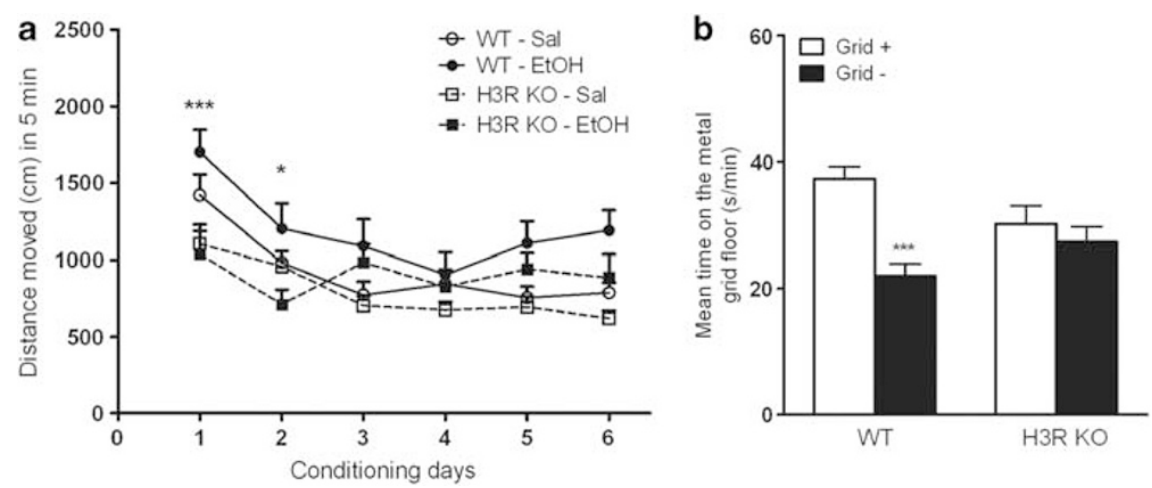

Figure 4 Mice lacking H3R do not develop alcohol-evoked place preference. (a) Activity of H3R KO and WT mice during conditioning sessions. Data present distance moved within a 5-min conditioning period following alcohol (2 g/ $/ \mathrm{kg})$ or saline injection. The WT C57BL/6) mice were significantly activated by alcohol on the first two days of conditioning; $* P<0.05$, *** $P<0.00$ I from the corresponding saline conditioning session, three-way ANOVA and Bonferroni post-test. (b) Time spent on the metal grid floor during a 15 -min preference test $24 \mathrm{~h}$ after the last conditioning session. Data present mean time spent (s/min + SEM) on the metal grid floor. White bars indicate the subgroup, which received alcohol paired with metal grid (Grid +) floor. Black bars indicate the subgroup where alcohol administration was paired with plastic floor (Grid-). Place preference was indexed by comparing the Grid + and Grid-groups ( $n=8$ for each subgroup). ${ }^{*} * * * 0.000$ I from the metal group (two-way ANOVA, Bonferroni post-test).

genotype $(\mathrm{F}=15.5, P<0.0001)$, and treatment $(\mathrm{F}=11.5$, $P<0.001$, Figure 4a). However, no interaction between these factors was found. Conditionings with alcohol resulted in a significant place preference in WT mice as indicated by a significant difference between the conditioning subgroups in the time spent on the metal grid floor $\left(\mathrm{F}_{3,27}=7.998\right.$, $P=0.0006$, one-way ANOVA, for WT Grid $+v s$ Grid - and, Tukey's post-test $n=8$ per subgroup, Figure $4 \mathrm{~b}$ ). The mice lacking H3R did not develop place preference which was confirmed by the lack of significant difference between the H3R KO Grid + and Grid- groups by one-way ANOVA plus Tukey post-test and a significant subgroup $\times$ genotype interaction $\left(\mathrm{F}_{1,27}=7.63, P<0.001\right)$ by two-way ANOVA. The activity of mice measured as distance moved during the preference test was similar between the genotypes: $2709 \pm 132.3 \mathrm{~cm}$ for WT and $3007 \pm 183.9 \mathrm{~cm}$ for H3R KO mice $(P=0.1947, t$-test $)$.

\section{Alcohol Does Not Stimulate H3R KO Mice}

The locomotor activity scores during habituation and in response to alcohol are shown in Figures $5 \mathrm{a}-\mathrm{c}$. The H3R KO mice were less active during the habituation period indicated by a significant genotype effect by twoway ANOVA $\left(\mathrm{F}_{1,29}=8.35, P=0.0072\right)$. A very short-lived ( $3 \mathrm{~min}$ ) activation by alcohol was present in WT mice in response to low alcohol dose $(1.5 \mathrm{~g} / \mathrm{kg})$, which was absent in H3R KO mice (Figure 5b). The depressant effect of high dose of alcohol was similar in both genotypes (Figure 5c).

\section{H3R KO Mice are Slightly Less Sensitive to Motor Incoordination by Alcohol}

The motor impairment by alcohol was tested using balance beam and rotarod tasks. The H3R KO mice were less sensitive to alcohol than the WT mice in the balance beam task after alcohol injection $(1.2 \mathrm{~g} / \mathrm{kg}$, i.p. $)$ as measured by the number of footslips. $(P=0.039, t$-test, Figure $5 \mathrm{~d})$. The $\mathrm{H} 3 \mathrm{R}$ KO mice learned to run on an accelerating rotarod similarly to their WT controls (Figure 5f) and there was no difference between the genotypes in the motor impairment induced by cumulative doses of alcohol $(1.5$ and $0.5 \mathrm{~g} / \mathrm{kg}$, Figure 5e). Following a sedative dose of alcohol $(4.0 \mathrm{~g} / \mathrm{kg}$, i.p.), the latency to LORR did not differ significantly between H3R KO and WT mice $(132 \pm 4 \mathrm{~s}$ vs $132 \pm 6 \mathrm{~s}$, $P>0.05)$. The sleep time, measured as the duration of LORR, did not differ significantly $(164.7 \pm 7.4 \mathrm{~min} v \mathrm{~s}$ $138.3 \pm 13.3 \mathrm{~min}, P>0.05)$.

\section{Genotype Difference in Dopamine and 5-HT Metabolism in Response to Alcohol Drinking}

Cortical catecholamine and metabolite concentrations in drug-naïve H3R KO mice are reported to be similar as those of the control animals (Toyota et al, 2002). The concentrations of dopamine, 5-HT, and noradrenaline and their metabolites were measured in mice immediately after the 12-day DID experiment (daily $4 \mathrm{~h}$ access to $20 \%$ alcohol). The H3R KO mice had lower striatal dopamine and 5-HT metabolite concentrations than the WT mice but the differences did not reach statistical significance (eg, DOPAC/DA ratio, $P=0.0673$, $t$-test, Table 1). A similar trend toward lower dopamine and 5-HT metabolite concentrations was found in prefrontal cortex in H3R KO mice. The concentration of DOPAC and 5-HIAA in H3R KO mice were significantly lower as compared with WT mice $(P=0.0418$ and $P=0.0224$, respectively, Table 1$)$.

\section{Plasma Alcohol Concentrations}

To determine whether the difference in alcohol consumption resulted in altered blood alcohol concentrations, blood samples were collected from $\mathrm{H} 3 \mathrm{R}$ KO and WT mice following $2 \mathrm{~g} / \mathrm{kg}$ (conditioning dose) and after the 4-h DID experiment. Alcohol concentrations in the plasma $5 \mathrm{~min}$ after alcohol injection were $28.3 \pm 1.6 \mathrm{mM} \quad(n=5)$ and $29.9 \pm 2.2 \mathrm{mM}(n=5)$ for WT and H3R KO mice, respectively. The difference was not significant ( $P=0.565, t$-test). We also measured the alcohol concentrations of H3R KO 
a

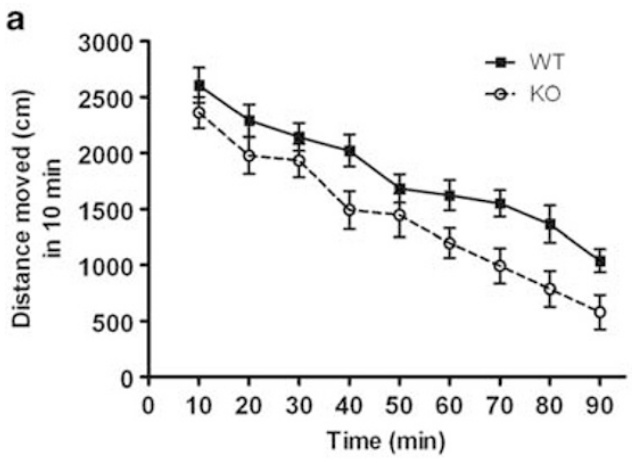

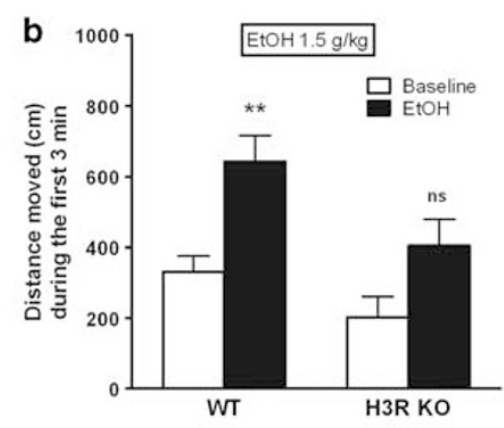

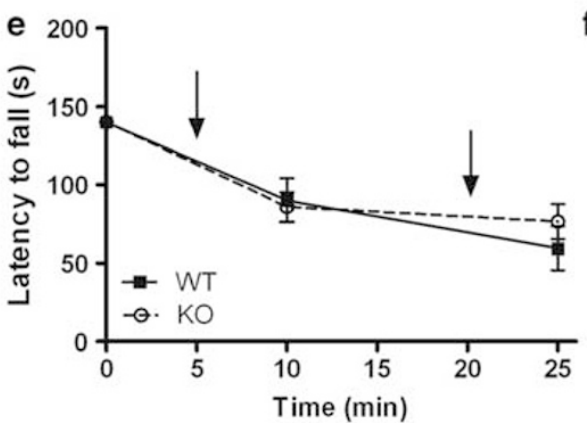

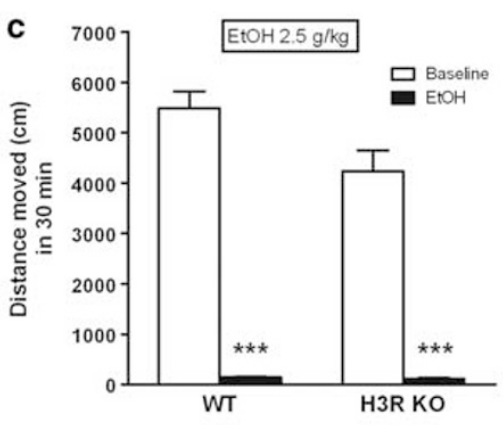

f

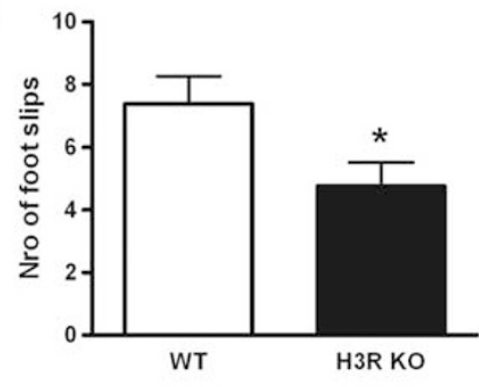

Figure 5 The $\mathrm{H} 3 \mathrm{R} \mathrm{KO}$ mice are tolerant to some but not all of the motor-impairing effects of alcohol. (a) Locomotor activity of H3R KO and WT mice during 90-min habituation period. The H3R KO mice were less active than the control animals $\left(F_{1,29}=8.35, P=0.0072\right.$, two-way RM ANOVA, $n=17$ $(\mathrm{WT}), n=14(\mathrm{H} 3 \mathrm{R} \mathrm{KO})$. (b) Cumulative locomotor activity during the first $3 \mathrm{~min}$ after alcohol injection ( $1.5 \mathrm{~g} / \mathrm{kg}$, i.p.) compared with the last $3 \mathrm{~min}$ of habituation (baseline). Only the WT mice are significantly stimulated by alcohol (** $P<0.0$, one-way ANOVA, $n=17(\mathrm{WT}), n=14(\mathrm{KO}), \mathrm{ns}=\mathrm{not}$ significant). (c) Locomotor activity during $30 \mathrm{~min}$ after alcohol injection $(2.5 \mathrm{~g} / \mathrm{kg}$, i.p.) compared with the last 30 min of habituation (**** $P<0.00$ I from baseline, one-way ANOVA). (d) Training performance on an accelerating rotarod (5-30 r.p.m., I 80 s) during 4 days. No genotype difference was detected in learning skills. $n=12$ for both genotypes. (e) Impairment of rotarod performance of H3R KO and WT mice after two cumulative alcohol injections ( 1.5 and $0.5 \mathrm{~g} / \mathrm{kg}$, i.p). Both injections were given 10 min before putting the mice on the accelerating rotarod (5-40 r.p.m.). No genotype difference was detected. $\mathrm{n}=12$ for both genotypes. $(\mathrm{f})$ The H3R KO mice perform better than the WT mice in the balance beam task after alcohol injection ( $1.2 \mathrm{~g} / \mathrm{kg}$, i.p.) as measured by the number of foot slips. $* P=0.039$, t- test, $n=8(\mathrm{WT}), n=12(\mathrm{KO})$.

Table I Concentrations of Striatal and Prefrontal Cortical Monoamine Neurotransmitters and their Metabolites in Male H3R KO and WT Mice in Response to Drinking in the Dark over 12 Days with an Access to Alcohol for 4-h Daily

\begin{tabular}{|c|c|c|c|c|}
\hline & \multicolumn{2}{|c|}{ Striatum } & \multicolumn{2}{|c|}{ Prefrontal cortex } \\
\hline & WT & H3R KO & WT & H3R KO \\
\hline DOPAC & $496.7 \pm 40.9$ & $393.4 \pm 41.7$ & $45.9 \pm 11.2$ & $\mathbf{I} \mathbf{8 . 8} \pm \mathbf{I . 9}(P=0.04 \mid 8)$ \\
\hline HVA & $603.7 \pm 46.3$ & $544.5 \pm 46.6$ & $84.6 \pm 10.1$ & $61.9 \pm 5.9$ \\
\hline $5-\mathrm{HT}$ & $153.0 \pm 17.4$ & $|43.0 \pm| 1.1$ & $259.4 \pm 6.8$ & $259.9 \pm 10.2$ \\
\hline $5-\mathrm{HIAA}$ & $103.1 \pm 8.0$ & $87.5 \pm 7.1$ & $83.6 \pm 4.9$ & $\mathbf{6 8 . 0} \pm \mathbf{3 . 1}(P=0.0224)$ \\
\hline 5-HIAA/5-HT & $0.2 \pm 0.04$ & $0.1 \pm 0.04(P=0.0673)$ & $0.8 \pm 0.2$ & $0.8 \pm 0.2$ \\
\hline Noradrenaline & $60.4 \pm 16.4$ & $50.9 \pm 9.7$ & $331.9 \pm 10.5$ & $331.9 \pm 4.4$ \\
\hline MHPG & $23.0 \pm 1.9$ & $23.0 \pm 0.8$ & $38.3 \pm 1.6$ & $37.0 \pm 1.4$ \\
\hline
\end{tabular}

Abbreviations: DA, dopamine; DOPAC, 3,4-dihydroxyphenylacetic acid; 5-HIAA, 5-hyrdroxy-indolacetic acid; 5-HT, 5-hydroxytryptamine; HVA, homovanillic acid; MHPG, 3-methoxy-4-hydroxyphenylglycol.

Values are means \pm SEM of $\mathrm{pg} / \mathrm{mg}$ tissue weight. Statistically significant values are shown with bold characters, $t$-test, $n=6$ per group.

and WT mice in the end of the DID experiment. The concentrations of alcohol after 4-h drinking were $6.0 \pm 0.3 \mathrm{mM}(n=6)$ for WT mice and $5.4 \pm 0.3 \mathrm{mM}(n=6)$ for H3R KO mice. There was no difference between WT and
KO mice in the amount of ethanol consumed (day 12, Figure $2 \mathrm{~b}$ ), nor was there a difference in the plasma ethanol concentrations between the two genotypes $(t=1.544$, $\mathrm{df}=10 ; P=0.154)$. 
We also measured the plasma alcohol concentrations following 2-h alcohol drinking in $\mathrm{C} 57 \mathrm{BL} / 6 \mathrm{~J}$ mice that received immepip $(30 \mathrm{mg} / \mathrm{kg}$ ) as a pretreatment. The plasma alcohol concentrations were $8.6 \pm 1.2 \mathrm{mM},(n=6)$ for saline controls and $17.7 \pm 5.5 \mathrm{mM},(n=5)$ for immepip-treated mice. The difference between the groups was significant ( $t=3.531, \mathrm{df}=9 ; P=0.0064)$ confirming the finding that immepip-treated mice drank more alcohol than the vehicletreated animals.

\section{DISCUSSION}

First, we report here that both genetic inactivation of $\mathrm{H} 3 \mathrm{R}$ and pharmacological treatment with an H3R antagonist suppress alcohol consumption in mice. Second, suppression of alcohol consumption in H3R KO mice is accompanied by a loss of CPP for alcohol. The lack of H3R does not result in robust changes in the motor-impairing effects of alcohol. However, alcohol-induced locomotor stimulation is diminished in H3R KO mice. Changes in alcohol kinetics are unlikely to explain our findings because the plasma alcohol concentrations were similar in H3R KO and WT mice. No marked differences in brain biogenic amine levels other than lower dopamine and 5-HT metabolite concentrations were detected in $\mathrm{H} 3 \mathrm{R}$ KO mice as compared with the WT mice.

Previously, we found that AA rats with a genetic preference for alcohol diminished their alcohol drinking following treatment with $\mathrm{H} 3 \mathrm{R}$ antagonists thioperamide and clobenprobit (Lintunen et al, 2001). Here, we show that mice lacking $\mathrm{H} 3 \mathrm{R}$ drink less alcohol in two different drinking models. Saccharin and quinine drinking were not different between genotypes suggesting that diminished alcohol consumption is not due to taste neophobia. Neither did the plasma alcohol concentrations differ between the genotypes indicative of similar alcohol kinetics. However, in the DID paradigm $\mathrm{H} 3 \mathrm{R} \mathrm{KO}$ mice were found to drink less sucrose solution compared with the WT controls. H3R KO mice consumed also less food than the controls. Neuronal histamine is known to regulate food intake and energy balance (Haas et al, 2008). Treatment with H3R antagonists suppresses food intake and decreases caloric intake, body weight, and plasma triglycerides in rodents (Malmlof et al, 2006) and in primates (Malmlof et al, 2007). Alcohol has a high caloric value so it is possible that $\mathrm{H} 3 \mathrm{R}$ KO mice drink less alcohol because their consumption of energy is lower. However, H3R ligands did not affect sucrose (high caloric value) drinking in the DID model suggesting that also other mechanisms such as diminished reward to alcohol might underlie the lower alcohol consumption in $\mathrm{H} 3 \mathrm{R} \mathrm{KO}$ mice and in animals treated with H3R antagonists. Neuronal histamine regulates also fluid intake and balance (Haas et al, 2008). Thus, another explanation to lower alcohol consumption in H3R KO mice could be that these mice are less thirsty. This was clearly not the case: the water consumption of H3R KO mice was similar to the controls and H3R ligands (ciproxifan and immepip) had a bidirectional effect on alcohol drinking without affecting sucrose drinking. Therefore, it is likely that a deficit in reward mechanisms is underlying the diminished alcohol drinking of H3R KO mice. Our data are also in agreement with the study of Munzar and colleagues (2004), which showed that H3R antagonists (acting as inverse agonists) thioperamide and clobenprobit reduce self-administration of amphetamine in rats.

The finding that H3R KO mice did not develop alcoholinduced CPP confirmed our hypothesis that H3R deletion affects brain reward mechanisms. We believe that this lack of alcohol reward is likely to contribute to the reduced alcohol consumption found in $\mathrm{H} 3 \mathrm{R}$ KO mice. In agreement with the present data, we reported earlier that $H 3 R$ antagonists ciproxifan and JNJ-10181457 inhibit CPP by alcohol in DBA/2J mice (Nuutinen et al, 2010b). The loss of CPP is interpreted as reduction of alcohol reward but it can also result from impairment in contextual learning and memory. However, H3R KO mice show normal learning behavior in a passive avoidance model (Toyota et al, 2002) and enhanced spatial learning and memory in a Barnes maze test (Rizk et al, 2004) suggesting that a stronger rather than total lack of CPP would have been a more expected outcome. Differences in CPP can also be due to different activity of the mouse groups during the preference test. Although the H3R KO mice were found to be less active than the controls when exploring a novel environment (alcohol stimulation study), the distance moved during the preference test was similar to control mice. Thus, the lack of alcohol-induced place preference was not due to different activity of $\mathrm{H} 3 \mathrm{R} \mathrm{KO}$ and WT mice.

The mice of C57BL/6J (B6) strain were initially described to display low or complete lack of alcohol-induced CPP (Cunningham et al, 1992). However, later studies have shown that the place preference can be induced to B6 mice by increasing the number of conditioning sessions with alcohol (Risinger et al, 2001; Boyce-Rustay and Risinger, 2003). Here, we used an extended protocol with six alcohol and six saline conditioning sessions, which resulted in a significant CPP in the WT animals.

In addition to lower alcohol drinking and alcohol CPP, the H3R KO mice were not stimulated by alcohol as were the control B6 mice. This was detected during the first 3-5 min following alcohol administration in place conditionings (EtOH dose $2 \mathrm{~g} / \mathrm{kg}$, two first days) and when a lower dose of alcohol $(1.5 \mathrm{~g} / \mathrm{kg})$ was used and horizontal activity was recorded for a longer period. In agreement with our finding, H3R KO mice are not stimulated by methamphetamine (Toyota et al, 2002; Okuda et al, 2009). The H3R KO did not differ from B6 control mice in rotarod task but made fewer mistakes as measured by number of foot slips on the balance beam test following alcohol administration. In line with this, we found previously that an H3R agonist increases alcohol-induced foot slips on the balance beam (Nuutinen et al, 2010a). Altogether these results suggest that lack of H3R function results in insensitivity toward the stimulating and to a lesser degree to the motor-impairing effect of alcohol.

To examine which neural circuits could underlie the altered alcohol-related behaviors in $\mathrm{H} 3 \mathrm{R}$ KO mice, we measured the tissue monoamines in striatum and prefrontal cortex. We found no significant alterations between the genotypes in dopamine, noradrenaline and 5-HT concentrations. The concentrations of dopamine metabolites DOPAC and 5-HIAA were lower in the prefrontal cortex in $\mathrm{H} 3 \mathrm{R} \mathrm{KO}$ mice and there was also a trend toward lower dopamine and 5-HT metabolite concentrations in striatum. 
In agreement with our results, Toyota et al (2002) found that the levels of dopamine, noradrenaline, and 5-HT were normal in cortical samples of drug-naïve H3R KO mice. However, H3R deletion had led to significantly lower histamine levels as compared with control mice. It would be important to study whether the release of histamine by alcohol is altered in H3R KO mice. If histamine mediates the alterations in alcohol consumption and alcohol reward in H3R KO mice found here, one would expect to see a difference in histamine release between the genotypes. On the basis of the suggested inhibitory role for histamine in reward (Brabant et al, 2010; Panula and Nuutinen, 2011), the alcohol-induced histamine release could be abnormal in $\mathrm{H} 3 \mathrm{R}$ KO mice. However, bearing in mind the heteroreceptor role of $\mathrm{H} 3 \mathrm{R}$ and the importance of dopamine in reward and reinforcement, it would be also important to study whether alcohol-induced dopamine release is altered in $\mathrm{H} 3 \mathrm{R} \mathrm{KO}$ mice. Especially because of the lack of CPP in H3R KO mice one would expect to find diminished dopamine release evoked by alcohol in nucleus accumbens of H3R KO mice. The H3R gene deletion might have also disrupted the suggested interaction of postsynaptic H3Rs with dopaminergic receptors in striatal GABAergic cells (Ferrada et al, 2008, 2009; Moreno et al, 2011) leading to different responses to alcohol in H3R and WT mice. Since only male mice were available for this study, the further studies should also involve females, because there may be sex differences in the observed behaviors.

In conclusion, using genetic and pharmacological approaches we demonstrate here that suppressed H3R function decreases alcohol consumption and alcohol reward in mice. Also the motor effects induced by alcohol are affected but to a lesser degree. Especially, the finding that alcohol intake can be suppressed by an H3R antagonist implies that the H3R antagonist currently in phase I-III clinical trials may have therapeutic potential in alcohol use disorders. Our results further suggest that in addition to influencing feeding, drinking, attentional, and cognitive functions, H3R has a role in reward processes.

\section{ACKNOWLEDGEMENTS}

We thank Johnson and Johnson Pharmaceutical Research and Development, LLC (San Diego, CA) for H3R KO mice and Professor Christopher Cunningham (Portland, OR) for advice in the CPP design. Ms Minna Mertanen is acknowledged for technical help. This study was financially supported by the Foundation for Alcohol Research and the Academy of Finland from grant numbers 118547 and 126744.

\section{DISCLOSURE}

PP has received lecture compensation from Abbott Laboratories. The remaining authors declare no conflict of interest.

\section{REFERENCES}

Alakarppa K, Tupala E, Mantere T, Sarkioja T, Rasanen P, Tarhanen J et al (2002). Effect of alcohol abuse on human brain histamine and tele-methylhistamine. Inflamm Res 51(Suppl 1): S40-S41.

Arrang JM, Garbarg M, Schwartz JC (1983). Auto-inhibition of brain histamine release mediated by a novel class (H3) of histamine receptor. Nature 302: 832-837.

Arrang JM, Garbarg M, Schwartz JC (1987). Autoinhibition of histamine synthesis mediated by presynaptic H3-receptors. Neuroscience 23: 149-157.

Arrang JM, Morisset S, Gbahou F (2007). Constitutive activity of the histamine H3 receptor. Trends Pharmacol Sci 28: 350-357.

Boyce-Rustay JM, Risinger FO (2003). Dopamine D3 receptor knockout mice and the motivational effects of ethanol. Pharmacol Biochem Behav 75: 373-379.

Brabant C, Alleva L, Grisar T, Quertemont E, Lakaye B, Ohtsu H et al (2009). Effects of the $\mathrm{H} 3$ receptor inverse agonist thioperamide on cocaine-induced locomotion in mice: role of the histaminergic system and potential pharmacokinetic interactions. Psychopharmacology (Berl) 202: 673-687.

Brabant C, Alleva L, Quertemont E, Tirelli E (2010). Involvement of the brain histaminergic system in addiction and addictionrelated behaviors: a comprehensive review with emphasis on the potential therapeutic use of histaminergic compounds in drug dependence. Prog Neurobiol 92: 421-441.

Brabant C, Charlier Y, Quertemont E, Tirelli E (2005). The H3 antagonist thioperamide reveals conditioned preference for a context associated with an inactive small dose of cocaine in C57BL/6J mice. Behav Brain Res 160: 161-168.

Brabant C, Quertemont E, Tirelli E (2006). Effects of the H3receptor inverse agonist thioperamide on the psychomotor effects induced by acutely and repeatedly given cocaine in C57BL/6J mice. Pharmacol Biochem Behav 83: 561-569.

Clapham J, Kilpatrick GJ (1994). Thioperamide, the selective histamine $\mathrm{H} 3$ receptor antagonist, attenuates stimulant-induced locomotor activity in the mouse. Eur J Pharmacol 259: 107-114.

Cunningham CL, Gremel CM, Groblewski PA (2006). Druginduced conditioned place preference and aversion in mice. Nat Protoc 1: 1662-1670.

Cunningham CL, Niehus DR, Malott DH, Prather LK (1992). Genetic differences in the rewarding and activating effects of morphine and ethanol. Psychopharmacology (Berl) 107: 385-393.

Ferrada C, Ferre S, Casado V, Cortes A, Justinova Z, Barnes C et al (2008). Interactions between histamine H3 and dopamine D2 receptors and the implications for striatal function. Neuropharmacology 55: 190-197.

Ferrada C, Moreno E, Casado V, Bongers G, Cortes A, Mallol J et al (2009). Marked changes in signal transduction upon heteromerization of dopamine $\mathrm{D} 1$ and histamine $\mathrm{H} 3$ receptors. $\mathrm{Br} \mathrm{J}$ Pharmacol 157: 64-75.

Galici R, Rezvani AH, Aluisio L, Lord B, Levin ED, Fraser I et al (2010). JNJ-39220675, a novel selective histamine $\mathrm{H}(3)$ receptor antagonist, reduces the abuse-related effects of alcohol in rats. Psychopharmacology (Berl) 214: 829-841.

Haas H, Panula P (2003). The role of histamine and the tuberomamillary nucleus in the nervous system. Nat Rev Neurosci 4: 121-130.

Haas HL, Sergeeva OA, Selbach O (2008). Histamine in the nervous system. Physiol Rev 88: 1183-1241.

Lintunen M, Hyytia P, Sallmen T, Karlstedt K, Tuomisto L, Leurs R et al (2001). Increased brain histamine in an alcohol-preferring rat line and modulation of ethanol consumption by $\mathrm{H}(3)$ receptor mechanisms. FASEB J 15: 1074-1076.

Lintunen M, Raatesalmi K, Sallmen T, Anichtchik O, Karlstedt K, Kaslin J et al (2002). Low brain histamine content affects ethanol-induced motor impairment. Neurobiol Dis 9: 94-105.

Lovenberg TW, Roland BL, Wilson SJ, Jiang X, Pyati J, Huvar A et al (1999). Cloning and functional expression of the human histamine H3 receptor. Mol Pharmacol 55: 1101-1107. 
Malmlof K, Golozoubova V, Peschke B, Wulff BS, Refsgaard HH, Johansen PB et al (2006). Increase of neuronal histamine in obese rats is associated with decreases in body weight and plasma triglycerides. Obesity (Silver Spring) 14: 2154-2162.

Malmlof K, Hastrup S, Wulff BS, Hansen BC, Peschke B, Jeppesen $\mathrm{CB}$ et al (2007). Antagonistic targeting of the histamine H3 receptor decreases caloric intake in higher mammalian species. Biochem Pharmacol 73: 1237-1242.

Moreno E, Hoffmann H, Gonzalez-Sepulveda M, Navarro G, Casado V, Cortes A et al (2011). Dopamine D1-histamine H3 receptor heteromers provide a selective link to the map-kinase signalling in gabaergic neurons of the direct striatal pathway. J Biol Chem 286: 5846-5854.

Munzar P, Nosal R, Goldberg SR (1998). Potentiation of the discriminative-stimulus effects of methamphetamine by the histamine $\mathrm{H} 3$ receptor antagonist thioperamide in rats. Eur $J$ Pharmacol 363: 93-101.

Munzar P, Tanda G, Justinova Z, Goldberg SR (2004). Histamine h3 receptor antagonists potentiate methamphetamine selfadministration and methamphetamine-induced accumbal dopamine release. Neuropsychopharmacology 29: 705-717.

Nuutinen S, Karlstedt K, Aitta-Aho T, Korpi ER, Panula P (2010a). Histamine and $\mathrm{H} 3$ receptor-dependent mechanisms regulate ethanol stimulation and conditioned place preference in mice. Psychopharmacology (Berl) 208: 75-86.

Nuutinen S, Vanhanen J, Pigni MC, Panula P (2010b). Effects of histamine $\mathrm{H} 3$ receptor ligands on the rewarding, stimulant and motor-impairing effects of ethanol in DBA/2J mice. Neuropharmacology 60: 1193-1199.
Okuda T, Zhang D, Shao H, Okamura N, Takino N, Iwamura T et al (2009). Methamphetamine- and 3,4-methylenedioxymethamphetamine-induced behavioral changes in histamine H3-receptor knockout mice. J Pharmacol Sci 111: 167-174.

Panula P, Nuutinen S (2011). Histamine and H3 receptor in alcohol-related behaviors. J Pharmacol Exp Ther 336: 9-16.

Pillot C, Heron A, Schwartz JC, Arrang JM (2003). Ciproxifan, a histamine $\mathrm{H} 3$-receptor antagonist/inverse agonist, modulates the effects of methamphetamine on neuropeptide mRNA expression in rat striatum. Eur J Neurosci 17: 307-314.

Pillot C, Ortiz J, Heron A, Ridray S, Schwartz JC, Arrang JM (2002). Ciproxifan, a histamine H3-receptor antagonist/inverse agonist, potentiates neurochemical and behavioral effects of haloperidol in the rat. J Neurosci 22: 7272-7280.

Rhodes JS, Best K, Belknap JK, Finn DA, Crabbe JC (2005). Evaluation of a simple model of ethanol drinking to intoxication in C57BL/6J mice. Physiol Behav 84: 53-63.

Risinger FO, Freeman PA, Greengard P, Fienberg AA (2001). Motivational effects of ethanol in DARPP-32 knock-out mice. J Neurosci 21: 340-348.

Rizk A, Curley J, Robertson J, Raber J (2004). Anxiety and cognition in histamine H3 receptor-/- mice. Eur J Neurosci 19: 1992-1996.

Schlicker E, Malinowska B, Kathmann M, Gothert M (1994). Modulation of neurotransmitter release via histamine $\mathrm{H} 3$ heteroreceptors. Fundam Clin Pharmacol 8: 128-137.

Toyota H, Dugovic C, Koehl M, Laposky AD, Weber C, Ngo K et al (2002). Behavioral characterization of mice lacking histamine H(3) receptors. Mol Pharmacol 62: 389-397. 